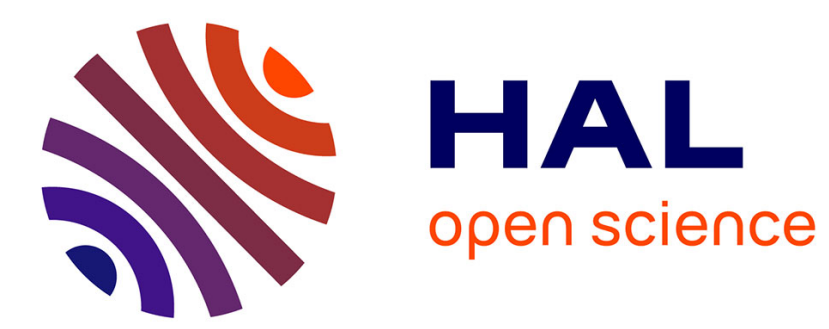

\title{
GENERAL REILLY-TYPE INEQUALITIES FOR SUBMANIFOLDS OF WEIGHTED EUCLIDEAN SPACES
}

Julien Roth

\section{To cite this version:}

Julien Roth. GENERAL REILLY-TYPE INEQUALITIES FOR SUBMANIFOLDS OF WEIGHTED

EUCLIDEAN SPACES. Colloquium Mathematicum, 2016, 144 (1), pp.127-136. hal-01140763

\section{HAL Id: hal-01140763 \\ https://hal.science/hal-01140763}

Submitted on 9 Apr 2015

HAL is a multi-disciplinary open access archive for the deposit and dissemination of scientific research documents, whether they are published or not. The documents may come from teaching and research institutions in France or abroad, or from public or private research centers.
L'archive ouverte pluridisciplinaire HAL, est destinée au dépôt et à la diffusion de documents scientifiques de niveau recherche, publiés ou non, émanant des établissements d'enseignement et de recherche français ou étrangers, des laboratoires publics ou privés. 


\title{
GENERAL REILLY-TYPE INEQUALITIES FOR SUBMANIFOLDS OF WEIGHTED EUCLIDEAN SPACES
}

\author{
JULIEN ROTH
}

\begin{abstract}
We prove new upper bounds for the first positive eigenvalue of a family of second order operators, including the Bakry-Émery Laplacian, for submanifolds of weighted Euclidean spaces.
\end{abstract}

\section{INTRODUCTION}

A weighted manifold $\left(\bar{M}, \bar{g}, \bar{\mu}_{f}\right)$ is a Riemannian manifold $(\bar{M}, \bar{g})$ endowed with a weighted volume form $\bar{\mu}_{f}=e^{-f} d v_{\bar{g}}$, where $f$ is a real-valued smooth function on $\bar{M}$ and $d v_{\bar{g}}$ is the Riemannian volume form associated with the metric $\bar{g}$. In the present note, we will focus on the case where $(\bar{M}, \bar{g})$ is the Euclidean space $\left(\mathbb{R}^{N}\right.$, can $)$ with its canonical flat metric and we will consider isometric immersions of Riemannian manifolds $\left(M^{n}, g\right)$ into $\left(\mathbb{R}^{N}\right.$, can $)$. For such an immersion, we define the weighted mean curvature vector $\mathbf{H}_{f}=\mathbf{H}-(\bar{\nabla} f)^{\perp}$, where $\mathbf{H}$ is the mean curvature vector of the immersion and $(\bar{\nabla} f)^{\perp}$ is the projection of $\bar{\nabla} f$ on the normal bundle $T^{\perp} M$.

We can define on $M$ a divergence and a Laplace operator associated with the volume form $\mu_{f}=e^{-f} d v_{g}$ by

$$
\operatorname{div}_{f} Y=\operatorname{div} Y-\langle\nabla f, Y\rangle \quad \text { and } \quad \Delta_{f} u=-\operatorname{div}_{f}(\nabla u)=\Delta u+\langle\nabla f, \nabla u\rangle,
$$

where $\nabla$ is the gradient on $M$, that is the projection on $T M$ of $\bar{\nabla}$. We call them the $f$ divergence and the $f$-Laplacian which is often called Bakry-Émery Laplacian, Witten Laplacian or drifting Laplacian in the litterature. It is a classical fact that $\Delta_{f}$ has a discrete spectrum composed of an infinite sequence of nonnegative real numbers

$$
0=\lambda_{0}<\lambda_{1} \leqslant \lambda_{2} \leqslant \cdots \longrightarrow+\infty .
$$

The eigenvalue $\lambda_{0}=0$ has multiplicity one and corresponds to constant functions. In [4], Batista, Cavalcante and Pyo proved the following upper bound for the first positive eigenvalue of $\Delta_{f}$ :

$$
\lambda_{1}\left(\Delta_{f}\right) \leqslant \frac{\int_{M}\left\|\mathbf{H}_{f}-\bar{\nabla} f\right\|^{2} \mu_{f}}{n \operatorname{Vol}_{f}(M)}=\frac{\int_{M}\left(\|\mathbf{H}\|^{2}+\|\nabla f\|^{2}\right) \mu_{f}}{n \operatorname{Vol}_{f}(M)},
$$

2010 Mathematics Subject Classification. 53C42, 53C21.

Key words and phrases. Weighted manifolds, Drifting Laplacian. 
where $\operatorname{Vol}_{f}(M)=\int_{M} \mu_{f}$ is the $f$-volume of $M$. This inequality is a weighted version of the classical Reilly inequality (see [9])

$$
\lambda_{1}(\Delta) \leqslant \frac{1}{n \operatorname{Vol}(M)} \int_{M}\|\mathbf{H}\|^{2} d v_{g} .
$$

Very recently, Domingo-Juan and Miquel [6] obtained the same inequality with a more complete characterization of the equality case by the use of mean curvature flow.

The aim of this note is to give a general inequality, which contains the above one, for a larger class of $f$-divergence-type operators. Precisely, for a positive symmetric divergence-free $(1,1)$-tensor $T$, we define the operator $L_{T, f}$ by

$$
L_{T, f} u=-\operatorname{div}_{f}(T \nabla u),
$$

for any $\mathcal{C}^{2}$ fonction $u$ on $M$. We prove the following theorem.

Theorem 1.1. Let $\left(M^{n}, g\right)$ be a connected and oriented closed Riemannian manifold isometrically immersed into the Euclidean space $\mathbb{R}^{N}$ endowed with a density $e^{-f}$. Let $S$ and $T$ be two symmetric divergence-free $(1,1)$-tensor over $M$. Assume moreover that $T$ is positive. Then, the first positive eigenvalue of the operator $L_{T, f}$ satisfies the following inequality

$$
\lambda_{1}\left(L_{T, f}\right)\left(\int_{M} \operatorname{tr}(S) \mu_{f}\right)^{2} \leqslant\left(\int_{M} \operatorname{tr}(T) \mu_{f}\right) \int_{M}\left(\left\|H_{S}\right\|^{2}+\|S \nabla f\|^{2}\right) \mu_{f} .
$$

Moreover, if equality holds in the case $S=\mathrm{Id}$ then $M$ is a self-shrinker for the mean curvature flow and $f_{\mid M}=a-\frac{c}{2} r_{p}^{2}$, where $r_{p}$ is the Euclidean distance to the center of mass $p$ of $M$. In particular, if $n=N-1$ and $H>0$ or $n=2, N=3$ and $M$ is embedded and has genus 0 , then $M$ a geodesic hypersphere.

As a corollary, we obtain a similar inequality for submanifolds of the sphere $\mathbb{S}^{N}$ which generalizes the corresponding inequality of [4] and [6] for the operator $L_{T, f}$ (see Corollary 4.4). We also prove a general non-weighted Reilly-type inequality (Theorem 5.1).

\section{PRELiminaries}

Let $\left(M^{n}, g\right)$ be a connected and oriented closed Riemannian manifold isometrically immersed into $\mathbb{R}^{N}$. We denote by $X$ its position vector, $B$ its second fundamental form and $\mathbf{H}=\operatorname{tr}(B)$ its mean curvature vector. For the case of hypersurfaces, we will also consider the real-valued mean curvature $H=\langle\mathbf{H}, \nu\rangle$, where $\nu$ is a unit normal vector field ( $H$ is defined up to a sign depend of the choice of $\nu$ ). We denote by $\left\{\partial_{1}, \cdots, \partial_{N}\right\}$ the canonical frame of $\mathbb{R}^{N}$ and for $k \in\{1, \cdots, N\}, X^{k}=\left\langle X, \partial_{k}\right\rangle$ the coordinate functions. We begin by giving the following elementary lemma. 
Lemma 2.1. If $A$ is a field of endomorphisms on $M$, we have

$$
\sum_{k=1}^{N}\left\langle A\left(\nabla X^{k}\right), \nabla X^{k}\right\rangle=\operatorname{tr}(A)
$$

Proof: Let $\left\{e_{1}, \cdots, e_{n}\right\}$ be a local orthonormal frame of $T M$. It is a classical fact that $\nabla X^{k}=\partial_{k}^{\top}=\sum_{i=1}^{n}\left\langle\partial_{k}, e_{i}\right\rangle e_{i}$. Hence, we have

$$
\begin{aligned}
\sum_{k=1}^{N}\left\langle A\left(\nabla X^{k}\right), \nabla X^{k}\right\rangle & =\sum_{k=1}^{N} \sum_{i, j=1}^{n}\left\langle\partial_{k}, e_{j}\right\rangle\left\langle\partial_{k}, e_{j}\right\rangle\left\langle A e_{i}, e_{j}\right\rangle \\
& =\sum_{i, j=1}^{n}\left(\sum_{k=1}^{N}\left\langle\partial_{k}, e_{j}\right\rangle\left\langle\partial_{k}, e_{j}\right\rangle\right)\left\langle A e_{i}, e_{j}\right\rangle \\
& =\sum_{i, j=1}^{n}\left\langle e_{i}, e_{j}\right\rangle\left\langle A e_{i}, e_{j}\right\rangle=\operatorname{tr}(A) .
\end{aligned}
$$

Note that, in particular, for $A=\mathrm{Id}$, we recover the well known identity $\sum_{k=1}^{N}\left\|\nabla X^{k}\right\|^{2}=n$.

Then, we recall briefly by some basic facts about the $f$-divergence. We first have the weighted version of the divergence theorem:

$$
\int_{M} \operatorname{div}_{f} Y \mu_{f}=0
$$

for any vector field $Y$ on $M$. From this, we deduce easily the integration by parts formula

$$
\int_{M} u \operatorname{div}_{f} Y \mu_{f}=-\int_{M}\langle\nabla u, X\rangle \mu_{f}
$$

for any smooth fonction $u$ and any vector field $Y$ on $M$.

Now, let $T$ be a divergence-free symmetric $(1,1)$-tensor. We associate with $T$ the second order differential operator $L_{T}$ defined by $L_{T} u:=-\operatorname{div}(T \nabla u)$, for any $\mathcal{C}^{2}$ function $u$ on $M$. We also associate with $T$ the following normal vector field:

$$
H_{T}=\sum_{i, j=1}^{n} T\left(e_{i}, e_{j}\right) B\left(e_{i}, e_{j}\right),
$$

where $\left\{e_{1}, \cdots, e_{n}\right\}$ is a local orthonormal frame of $T M$. We also defined a corresponding weighted operator by $L_{T, f} u=-\operatorname{div}_{f}(T \nabla u)$ for any $\mathcal{C}^{2}$ function $u$. We have the following weighted Hsiung-Minkowski formula. 
Lemma 2.2. We have

$$
\int_{M}\left(\left\langle X, H_{T}-T \nabla f\right\rangle+\operatorname{tr}(T)\right) \mu_{f}=0 .
$$

Proof: First, it is well known that $L_{T} X=-H_{T}$. The proof of this fact is standard and completely analogue to the case $T=\mathrm{Id}$, that is, $\Delta X=-n \mathbf{H}$ and uses the fact that $\operatorname{div}(T)=0$. From this, we deduce

$$
\begin{aligned}
L_{T}\|X\|^{2} & =\sum_{k=1}^{N} L_{T}\left(\left(X^{k}\right)^{2}\right) \\
& =-2 \sum_{k=1}^{N} \operatorname{div}\left(X^{k} T\left(\nabla X^{k}\right)\right) \\
& =2 \sum_{k=1}^{N}\left(X^{k} L_{T} X^{k}-\left\langle\nabla X^{k}, T\left(\nabla X^{k}\right)\right\rangle\right) \\
& =-2\left\langle X, H_{T}\right\rangle-2 \operatorname{tr}(T),
\end{aligned}
$$

where we have used $L_{T} X=-H_{T}$ and Lemma 2.1 for the last line. Therefore, we get

$$
\begin{aligned}
\frac{1}{2} L_{T, f}\|X\|^{2} & =\frac{1}{2} L_{T}\|X\|^{2}+\frac{1}{2}\left\langle T\left(\nabla\|X\|^{2}\right), \nabla f\right\rangle \\
& =-\left\langle X, H_{T}\right\rangle-\operatorname{tr}(T)+\frac{1}{2}\left\langle\nabla\|X\|^{2}, T \nabla f\right\rangle, \\
& =-\left\langle H_{T}-T \nabla f, X\right\rangle-\operatorname{tr}(T)
\end{aligned}
$$

where we have used (4), the symmetry of $T$ and the fact that $\nabla\|X\|^{2}=2 X^{\top}$. We conclude by integrating over $M$ for the measure $\mu_{f}$ and using the fact that $\int_{M} L_{T, f}\|X\|^{2} \mu_{f}=0$ by (1).

We can obtain a weighted Hsiung-Minkowski inequality by the use of the operator $L_{T, f}$. Namely, we prove the following lemma.

\section{Proof of Theorem 1.1}

Now, we have all the ingredients to prove the main theorem of this note. First, since we assume that the tensor $T$ is positive, the operator $L_{T, f}$ has a discrete nonnegative spectrum. The first eigenvalue is $\lambda_{0}=0$ is of mulitplicity one and the associated eigenfunctions are the constants. Thus, we denote by $\lambda_{1}\left(L_{T, f}\right)$ its first positive eigenvalue. From the definition of $L_{T, f}$ and (2) we have the following the variational characterization of $\lambda_{1}\left(L_{T, f}\right)$

$$
\lambda_{1}\left(L_{T, f}\right)=\inf \left\{\frac{\int_{M}\langle T \nabla u, \nabla u\rangle \mu_{f}}{\int_{M} u^{2} \mu_{f}} \mid u \in \mathcal{C}^{\infty}(M), \int_{M} u \mu_{f}=0\right\} .
$$


Up to a translation if needed, we may assume that the $\mu_{f}$-center of mass of $M$ is zero, that is, $\int_{M} X \mu_{f}=\overrightarrow{0}$. Hence, the coordinates can be used as test functions in the Rayleigh quotient and we have

$$
\lambda_{1}\left(L_{T, f}\right) \int_{M}\|X\|^{2} \mu_{f} \leqslant \int_{M} \sum_{i=1}^{N}\left\langle T \nabla X^{k}, X^{k}\right\rangle \mu_{f},
$$

which gives, by Lemma 2.1 ,

$$
\lambda_{1}\left(L_{T, f}\right) \int_{M}\|X\|^{2} \mu_{f} \leqslant \int_{M} \operatorname{tr}(T) \mu_{f} .
$$

Now, we have

$$
\begin{aligned}
\lambda_{1}\left(L_{T, f}\right)\left(\int_{M} \operatorname{tr}(S) \mu_{f}\right)^{2} & \leqslant \lambda_{1}\left(L_{T, f}\right)\left(\int_{M}\left(\left\langle X, H_{S}-S \nabla f\right\rangle\right) \mu_{f}\right)^{2} \\
& \leqslant \lambda_{1}\left(L_{T, f}\right)\left(\int_{M}\|X\|^{2} \mu_{f}\right)\left(\int_{M}\left\|H_{S}-S \nabla f\right\|^{2} \mu_{f}\right) \\
& \leqslant\left(\int_{M} \operatorname{tr}(T) \mu_{f}\right)\left(\int_{M}\left\|H_{S}-S \nabla f\right\|^{2} \mu_{f}\right),
\end{aligned}
$$

where we have used succesively the weighted Hsiung-Minkowski formula, the Cauchy-Schwarz inequality and (4). Since $H_{S}$ is normal and $S \nabla f$ is tangent to $M$, we get the wanted upper bound

$$
\lambda_{1}\left(L_{T, f}\right)\left(\int_{M} \operatorname{tr}(S) \mu_{f}\right)^{2} \leqslant\left(\int_{M} \operatorname{tr}(T) \mu_{f}\right) \int_{M}\left(\left\|H_{S}\right\|^{2}+\|S \nabla f\|^{2}\right) \mu_{f} .
$$

Equality case. Now, we assume that $S=\mathrm{Id}$. Then, the inequality becomes

$$
\lambda_{1}\left(L_{T, f}\right) \leqslant\left(\int_{M} \operatorname{tr}(T) \mu_{f}\right) \int_{M}\left(\|\mathbf{H}\|^{2}+\|\nabla f\|^{2}\right) \mu_{f} .
$$

If, equality occurs then all the above inequalities are equalities. In particular, equality occurs in the Cauchy-Schwarz inequality and we have $\mathbf{H}-\nabla f=c X$ for some constant $c$. Identifying tangential and normal parts, we get $\nabla f=-c X^{\top}$ and $\mathbf{H}=c X^{\perp}$.

The normal equation $\mathbf{H}=c X^{\perp}$ is exactly the definition of a self-similar solution of the mean curvature flow. Since $M$ is a compact submanifold of $\mathbb{R}^{N}, c$ cannot be zero. The case $c>0$ is no more possible. Indeed, if $c>0$, then $M$ is a self-expander, but it is well known that there exists no compact self-expander. Hence, the only possibility is $c<0$, that is $M$ is a self-shrkiner.

In addition, since $X^{\top}=\frac{1}{2} \nabla\|X\|^{2}$, the tangential equation becomes $\nabla\left(f+\frac{c}{2}\|X\|^{2}\right)=0$. Since $M$ is connected, there exists a constant $a$ such that $f_{\mid M}=a-\frac{c}{2}\|X\|^{2}$.

In the particular cases $N=n-1$ and $H>0$ or $n=2, N=3$ and $M$ is embedded 
and has genus 0 , then we know from [8] and [5] respectively that $M$ has to be a geodesic hypersphere. This finishes the proof of the equality case.

\section{SOME COROLLARIES}

In this secction, we state some corollaries obtained from Theorem 1.1. The first corollary is just a particular case of Theorem 1.1 invovling higher order mean curvatures. We before stating it, we recall briefely the definition of higher order mean curvatures and their associated tensors. For $r \in\{1, \cdots, n\}$, we set

$$
T_{r}=\frac{1}{r !} \sum_{\substack{i, i_{1}, \cdots, i_{r} \\
j, j_{1}, \cdots, j_{r}}} \epsilon\left(\begin{array}{l}
i, i_{1}, \cdots, i_{r} \\
j, j_{1}, \cdots, j_{r}
\end{array}\right)\left\langle B_{i_{1} j_{1}} B_{i_{2} j_{2}}\right\rangle \cdots\left\langle B_{i_{r-1} j_{r-1}} B_{i_{r} j_{r}}\right\rangle e_{i}^{*} \otimes e_{j}^{*},
$$

if $r$ is even and

$$
T_{r}=\frac{1}{r !} \sum_{\substack{i, i_{1}, \cdots, i_{r} \\
j, j_{1}, \cdots, j_{r}}} \epsilon\left(\begin{array}{l}
i, i_{1}, \cdots, i_{r} \\
j, j_{1}, \cdots, j_{r}
\end{array}\right)\left\langle B_{i_{1} j_{1}} B_{i_{2} j_{2}}\right\rangle \cdots\left\langle B_{i_{r-1} j_{r-1}} B_{i_{r} j_{r}}\right\rangle B_{i_{r}, j_{r}} \otimes e_{i}^{*} \otimes e_{j}^{*} \text {, }
$$

where the $B_{i j}$ 's are the coefficients of the second fundamental form $B$ in a local orthonormal frame $\left\{e_{1}, \cdots, e_{n}\right\}$ and $\epsilon$ is the standard signature for permutations. Here, $\left\{e_{1}^{*}, \cdots, e_{n}^{*}\right\}$ is the dual coframe of $\left\{e_{1}, \cdots, e_{n}\right\}$. By definition, the $r$-th mean curvature is $H_{r}=\frac{1}{c(r)} \operatorname{tr}\left(T_{r}\right)$, where $c(r)=(n-r)\left(\begin{array}{l}r \\ n\end{array}\right)$. Note that $H_{r}$ is a real function if $r$ is even and a normal vector field if $r$ is odd. By convention, we set $H_{0}=1$. Moreover, always if $r$ is even, we show easily that $H_{T_{r}}=c(r) H_{r+1}$, where $H_{T_{r}}$ is given by the relation (3).

In the case of hypersurfaces, we can consider the higher order mean curvatures as scalar functions also for odd indices by taking $B$ as the real-valued second fundamental form.

By the symmetry of $B$, these tensors are clearly symmetric. Morover, we have the following well-known lemma (the proof of this lemma can be found in [7] for instance).

Lemma 4.1. (1) If $n=N-1$, then for any $r \in\{0, \cdots, n-1\}$, we have $\operatorname{div}\left(T_{r}\right)=$ 0 .

(2) If $n \leqslant N-2$, then for any even $r \in\{0, \cdots, n-1\}$, we have $\operatorname{div}\left(T_{r}\right)=0$.

The tensor $T_{r}$ is the linearized operator associated with the $r$-th mean curvature and plays a crucial role in the study of the $r$-stability of hypersurfaces with constant $r$-th mean curvature (see [1] for instance).

We can state the following corollary obtained immediately form Theorem 1.1 . 
since the tensors $T_{r}$ are divergence-free. Note that this corollary is a weighted version of an inequality of Alias and Malacarne [2].

Corollary 4.2. Let $\left(M^{n}, g\right)$ be a connected and oriented closed Riemannian manifold isometrically immersed into the Euclidean space $\mathbb{R}^{N}$ endowed with a density $e^{-f}$. Let $r, s \in\{1, \cdots, n-1\}$. Assume that $r$ and $s$ are even if $N>n-1$ and assume moreover that $T_{r}$ is positive. Then, the first positive eigenvalue of the operator $L_{r, f}=L_{T_{r}, f}$ satisfies the following inequality

$$
\lambda_{1}\left(L_{r, f}\right)\left(\int_{M} H_{s} \mu_{f}\right)^{2} \leqslant \frac{c(r)}{c(s)}\left(\int_{M} H_{r} \mu_{f}\right) \int_{M}\left(c(s)^{2}\left\|H_{s+1}\right\|^{2}+\left\|T_{s} \nabla f\right\|^{2}\right) \mu_{f} .
$$

Remark 4.3. In the case of hypersurfaces, it is sufficient to have $H_{r+1}>0$ to ensure that $T_{r}$ is positive (see [3] for instance).

Now, using the embedding of the sphere $\mathbb{S}^{N}$ into the Euclidean space $\mathbb{R}^{N+1}$, we can prove this second corollary for submanifolds of the sphere $\mathbb{S}^{N}$. Precisely, we have the following result.

Corollary 4.4. Let $\left(M^{n}, g\right)$ be a connected and oriented closed Riemannian manifold isometrically immersed into the sphere $\mathbb{S}^{N}$ endowed with a density $e^{-f}$. Let $S$ and $T$ be two symmetric divergence-free $(1,1)$-tensor over $M$. Assume moreover that $T$ is positive. Then, the first positive eigenvalue of the operator $L_{T, f}$ satisfies the following inequality

$$
\lambda_{1}\left(L_{T, f}\right)\left(\int_{M} \operatorname{tr}(S) \mu_{f}\right)^{2} \leqslant\left(\int_{M} \operatorname{tr}(T) \mu_{f}\right) \int_{M}\left(\left\|H_{S}\right\|^{2}+\operatorname{tr}(S)^{2}+\|S \nabla f\|^{2}\right) \mu_{f} .
$$

Proof: The proof comes easily form Theorem 1.1. We denote by $\phi$ the immersion of $M$ into $\mathbb{S}^{N}$ and we consider the canonical immersion $i$ of $\mathbb{S}^{N}$ into $\mathbb{R}^{N+1}$ and we extend the weight $f$ defined on $\mathbb{S}^{N}$ to a weight $\widetilde{f}$ on $\mathbb{R}^{N+1}$, for instance by taking $\widetilde{f}(x)=|x| f\left(\frac{x}{|x|}\right)$ for any $x \in \mathbb{S}^{N}$ and $\widetilde{f}(0)=0$. From Theorem 1.1 we have

$$
\lambda_{1}\left(L_{T, f}\right)\left(\int_{M} \operatorname{tr}(S) \mu_{f}\right)^{2} \leqslant\left(\int_{M} \operatorname{tr}(T) \mu_{f}\right) \int_{M}\left(\left|H_{S}^{\prime}\right|^{2}+|S \nabla \widetilde{f}|^{2}\right) \mu_{f},
$$

where $H_{S}^{\prime}$ is defined by $H_{S}=\sum_{i, j=1}^{n} S\left(e_{i}, e_{j}\right) B^{\prime}\left(e_{i}, e_{j}\right)$ with $B^{\prime}$ the second fundamental form of the immersion of $M$ into $\mathbb{R}^{N+1}$. Obviously, the second fundamental forms $B$ of $\phi$ and $B^{\prime}$ of $i \circ \phi$ are linked by the relation $B^{\prime}=B-g \phi$. Hence, we get immediately $H_{S}^{\prime}=H_{S}-\operatorname{tr}(S) \phi$. Therefore, we deduce that $\left\|H_{S}^{\prime}\right\|^{2}=\left\|H_{S}\right\|^{2}+\operatorname{tr}(S)^{2}$, since $H_{S}$ and $\phi$ are orthogonal and $\|\phi\|=1$ since $M$ is contained in the sphere $\mathbb{S}^{N}$. Reporting this in (5), and since $f$ coincides with $\widetilde{f}$ on $M$, we have $\nabla \widetilde{f}=\nabla f$ and so

$$
\lambda_{1}\left(L_{T, f}\right)\left(\int_{M} \operatorname{tr}(S) \mu_{f}\right)^{2} \leqslant\left(\int_{M} \operatorname{tr}(T) \mu_{f}\right) \int_{M}\left(\left\|H_{S}\right\|^{2}+\operatorname{tr}(S)^{2}+\|S \nabla f\|^{2}\right) \mu_{f} .
$$


This concludes the proof.

For submanifolds of spheres, we have immediately the following corollary involving higher order mean curvatures.

Corollary 4.5. Let $\left(M^{n}, g\right)$ be a connected, oriented closed Riemannian manifold isometrically immersed into the sphere $\mathbb{S}^{N}$ endowed with a density $e^{-f}$. Let $r, s \in$ $\{1, \cdots, n-1\}$. Assume that $r$ and $s$ are even if $N>n-1$ and assume moreover that $T_{r}$ is positive. Then, the first eigenvalue of the operator $L_{r, f}$ satisfies the following inequality

$$
\lambda_{1}\left(L_{r, f}\right)\left(\int_{M} H_{s} \mu_{f}\right)^{2} \leqslant \frac{c(r)}{c(s)}\left(\int_{M} H_{r} \mu_{f}\right) \int_{M}\left(c(s)^{2}\left\|H_{s+1}\right\|^{2}+c(s)^{2} H_{s}^{2}+\left\|T_{s} \nabla f\right\|^{2}\right) \mu_{f} .
$$

\section{A GENERAL NON-WEIGHTED INEQUALITY}

In the classical case, that is, without density, the equality case can be characterized in a more rigid way. Namely, we have the following result

Theorem 5.1. Let $\left(M^{n}, g\right)$ be a connected, oriented closed Riemannian manifold isometrically immersed into $\mathbb{R}^{N}$. Assume that $M$ is endowed with two symmetric and divergence-free $(1,1)$-tensors $S$ et $T$. Assume in addition that $T$ is positive definite. Then, the first positive eigenvalue of the operator $L_{T}$ satisfies

$$
\lambda_{1}\left(L_{T}\right)\left(\int_{M} \operatorname{tr}(S) d v_{g}\right)^{2} \leqslant\left(\int_{M} \operatorname{tr}(T) d v_{g}\right)\left(\int_{M}\left\|H_{S}\right\|^{2} d v_{g}\right) .
$$

Moreover, if $N>n-1$ and $H_{S}$ does not vanish identically and equality occurs, then $\operatorname{tr}(S)$ and $\left\|H_{S}\right\|$ are non-zero constants and $M$ is $S$-minimally immersed into a geodesic hypersphere of $\mathbb{R}^{N}$ of radius $\frac{|\operatorname{tr}(S)|}{\left\|H_{S}\right\|}$.

In particular, if $n=N-1$ and $H_{S}$ does not vanish identically then if equality holds, then $\operatorname{tr}(S)$ and $H_{S}$ are non-zero constants and $M$ is a geodesic hypersphere of radius $\frac{|\operatorname{tr}(S)|}{\left|H_{S}\right|}$.

Remarks 5.2. (1) Note that for this theorem, contrary to Theorem 1.1 we do not need to assume that $M$ is embedded to characterize the equality case, the embedding is obtained as a consequence.

(2) For $T=$ Id, we have

$$
\lambda(\Delta)\left(\int_{M} \operatorname{tr}(S) d v_{g}\right)^{2} \leqslant n \operatorname{Vol}(M)\left(\int_{M}\left\|H_{S}\right\|^{2} d v_{g}\right),
$$

which was proved by Grosjean in [7].

Proof: The inequality is immediate form Theorem 1.1 with $f$ identically zero. If equality occurs, then all the above inequalities in the proof of Theorem 1.1 become equalities. In particular, we have $H_{S}=c X$ from the equality case of Cauchy-Schwarz 
inequality, where $c$ is a non-zero constant. This means that the position vector $X$ is everywhere normal to $M$. But, on the other hand, since $\nabla\|X\|^{2}=2 X^{\top}$, we get that $\nabla\|X\|^{2}=0$. Hence, since $M$ is connected, then $\|X\|=r$ is constant and $M$ lies in a geodesic hypersphere of radius $r$. Moreover, since $H_{S}=c X$, we get that $\left\|H_{S}\right\|$ is also constant and from Equation (4), we conclude that $\operatorname{tr}(S)=-\left\langle X, H_{S}\right\rangle=-\frac{1}{c}\left\|H_{S}\right\|^{2}$. Thus, $\operatorname{tr}(S)$ is also constant. Note that, since we assume that $H_{S}$ does not vanish identically, $\operatorname{tr}(S)$ and $\left\|H_{S}\right\|$ are non-zero constants and we have $r=\frac{|\operatorname{tr}(S)|}{\left\|H_{S}\right\|}$.

Now, we will show that the immersion of $M$ in this hypersphere $\mathbb{S}^{N-1}(r)$ is $S$-minimal, that is, $\widetilde{H}_{S}=0$, where is defined by

$$
H_{S}=\sum_{i, j=1}^{n} S\left(e_{i}, e_{j}\right) \widetilde{B}\left(e_{i}, e_{j}\right),
$$

with $\widetilde{B}$ the second fundamental form of $M$ in $\mathbb{S}^{N-1}(r)$. Clearly, we have $B=\widetilde{B}+\bar{B}$ where $\bar{B}$ is the second fundamental form of $\mathbb{S}^{N-1}$ into $\mathbb{R}^{N}$ and is given by $\bar{B}_{i j}=$ $-\frac{1}{r^{2}} \delta_{i j} X$. From this fact and the definition of $H_{S}$ and $\widetilde{H}_{S}$, we get

$$
\begin{aligned}
H_{S} & =\widetilde{H}_{S}-\frac{1}{r^{2}} \sum_{i, j}^{n} S\left(e_{i}, e_{j}\right) \delta_{i j} X \\
& =\widetilde{H}_{S}-\frac{1}{r^{2}} \operatorname{tr}(S) X \\
& =\widetilde{H}_{S}-\frac{\left|H_{S}\right|^{2}}{\operatorname{tr}(S)} X \\
& =\widetilde{H}_{S}+c X=\widetilde{H}_{S}+H_{S} .
\end{aligned}
$$

We deduce that $\widetilde{H}_{S}=0$, that is $M$ is $S$-minimally immersed into $\mathbb{S}^{N-1}(r)$.

If $n=N-1$, if equality occurs, by the above discussion and since $M$ has no boundary, then $M$ is $\mathbb{S}^{N-1}(r)$. This concludes the proof.

\section{REFERENCES}

[1] H. Alencar, M.P. Do Carmo \& H. Rosenberg, On the first eigenvalue of Linearized operator of the $r$-th mean curvature of a hypersurface, Ann. Glob. Anal. Geom., 11 (1993), 387-395.

[2] L.J. Alias \& J.M. Malacarne, On the first eigenvalue of the linearized operator of the higher order mean curvature for closed hypersurfaces in space forms, Illinois J. Math. 48 (1) (2004) 219240.

[3] J.L.M. Barbosa \& A.G. Colares, Stability of hypersurfaces with constant r-mean curvature, Ann. Global Anal. Geom. 15 (1997), 277-297.

[4] M. Batista, M.P. Cavalcante \& J. Pyo, Some isomperimetric inequalities and eigenvalue estimates in weighted manifolds, J. Math. Anal. Appl., 419(1) (2014), 617-626.

[5] S. Brendle, Embedded self-similar shrinkers of genus 0, arXiv:1411.4640

[6] M.C. Domingo-Juan \& V. Miquel, Reilly's type inequality for the Laplacian associated to a density related with shrinkers for MCF, arXiv:1503.01332.

[7] J.F. Grosjean, Upper bounds for the first eigenvalue of the Laplacian on compact manifolds, Pac. J. Math. 206(1) (2002) 93-111. 
[8] G. Huisken, Asymptotic behavior for singularities of the mean curvature flow, J. Differential Geom. 31 (1990), 285-299.

[9] R.C. Reilly, On the first eigenvalue of the Laplacian for compact submanifolds of Euclidean space, Comment. Math. Helv. 52 (1977), 525-533.

(J. Roth) LAMA, UPEM-UPEC-CNRS, Cité Descartes, Champs Sur MARne, 77454 MARNE-LA-VALLÉE CEDEX 2, FRANCE

E-mail address: julien.roth@u-pem. fr 